Published in final edited form as:

Expert Rev Gastroenterol Hepatol. 2016 ; 10(2): 161-163. doi:10.1586/17474124.2016.1125290.

\title{
Circulating microRNAs in nonalcoholic fatty liver disease
}

\author{
Johanna K. DiStefano ${ }^{1}$ and Glenn S. Gerhard ${ }^{2}$ \\ Johanna K. DiStefano: distefanoj@NJHealth.org; Glenn S. Gerhard: gsgerhard@temple.edu \\ ${ }^{1}$ Center for Genes, Environment, and Health, National Jewish Health, 1400 Jackson Street, \\ Denver, CO 80206, Phone: 303-398-2357 \\ ${ }^{2}$ Department of Medical Genetics and Molecular Biochemistry, Temple University School of \\ Medicine, 3500 N. Broad Street, Philadelphia, PA 19140, Phone: 215-707-5415
}

\section{Summary}

Liver biopsy is currently recognized as the most accurate method for diagnosing and staging nonalcoholic fatty liver disease (NAFLD). However, this procedure is typically performed when disease has progressed to clinically significant stages, thereby limiting early diagnosis of patients who are at high risk for development of liver- and cardiovascular-related morbidity and mortality. Recently, microRNAs (miRNAs), short, noncoding RNAs that regulate gene expression, have been associated with histological features of NAFLD and are readily detected in the circulation. As such, miRNAs are emerging as potentially useful noninvasive markers with which to follow the progression of NAFLD. In this article, we present the evidence linking circulating miRNAs with NAFLD and discuss the potential value of circulating miRNA profiles in the development of improved methods for NAFLD diagnosis and clinical monitoring of disease progression.

\section{Keywords}

miRNA; NAFLD; NASH; liver fibrosis; biomarker; epigenetics

\begin{abstract}
Nonalcoholic fatty liver disease (NAFLD) encompasses a spectrum of conditions ranging from steatosis to nonalcoholic steatohepatitis (NASH), and can progress to fibrosis, cirrhosis, and hepatocellular carcinoma, all of which are associated with increased morbidity and mortality '. A number of clinical factors have been associated with NAFLD including insulin resistance, type 2 diabetes (T2D), iron metabolism, and others . Currently, histological examination is considered the reference standard for the diagnosis and staging of liver fibrosis. However, a number of drawbacks to this procedure, including patient discomfort and risk for serious complications, sampling error and bias, variability in histopathologic interpretation, and significant financial cost, have led to a growing interest in alternative noninvasive strategies for the evaluation of NAFLD .
\end{abstract}

\footnotetext{
Correspondence to: Johanna K. DiStefano, distefanoj@NJHealth.org.
}

Financial and competing interests disclosure: The authors have no relevant affiliations or financial involvement with any organization or entity with a financial interest in or financial conflict with the subject matter or materials discussed in the manuscript. This includes employment, consultancies, honoraria, stock ownership or options, expert testimony, grants or patents received or pending, or royalties. 
Recently, microRNAs (miRNAs), a class of noncoding RNAs whose primary intracellular role is to modulate gene expression, have emerged as novel molecules involved in a variety of disease processes. MiRNAs are abundantly expressed in the liver, where they regulate a diverse array of functions ', and play a key role in the pathogenesis of many liver diseases including chronic viral hepatitis, drug-related and alcoholic liver toxicity, autoimmune hepatitis, hepatic fibrosis, and hepatocellular carcinoma'. MiRNAs can also circulate freely in the blood protected from RNase-dependent degradation under most physiological conditions . Cells release miRNAs through both passive and active mechanisms. For example, increased cell death, as occurs in NAFLD via ballooning degeneration, can release miRNAs into the circulation, where they are protected from degradation in part through binding to the Argonaute-2 protein, or packaged in high density lipoprotein particles . However, most circulating miRNAs are secreted from cells through active energy-dependent processes via storage in microvesicles (i.e, exosomes, microparticles, and shedding vesicles). The regulation and functional roles of microvesicular miRNAs are areas of active investigation in many diseases, including NAFLD . The relatively stable nature of circulating miRNAs, combined with the non-invasive manner in which they can be measured, has positioned miRNAs as potential biomarkers for NAFLD.

In addition to a potential mechanistic role described elsewhere, the multicellular nature and pathophysiological progression of NAFLD suggest that miRNAs may be associated with different disease stages. In a study of 84 circulating miRNAs measured in 47 NASH patients, 30 individuals with simple steatosis, and 19 healthy controls, levels of miR-122, miR-192, and miR-375 were upregulated in patients with NASH compared to those with simple steatosis, and were associated with histological disease severity . However, only serum levels of miR-122 were significantly associated with advanced fibrosis in comparisons of F0-1 and F2-3, consistent with other studies of NAFLD in humans and animal models '. The accuracy of miR-122, miR-192, and miR-375 to discriminate advanced from mild disease was fair (area under the receiver operating characteristic curve or AUC $=\sim 0.7$ ). In a cross-comparison analysis with NAFLD biomarker CK18 , , miR-122 was able to predict NASH and fibrosis with greater sensitivity, and both serum miR-122 and miR-192 levels were positively correlated with serum CK18 concentration $(\mathrm{P}<0.03)$. Given that miR-122 is the most abundantly expressed miRNA in liver, its correlation with an apoptotic marker such as CK18 is not surprising.

A number of studies focusing on a specific miRNA or miRNA panel have also been recently published. In a study of 34 individuals with NAFLD and 19 healthy controls, blood levels of miR-122, miR-34a, and miR-16 were significantly higher in NAFLD patients, although levels of miR-21, reportedly dysregulated in NAFLD, were not different between the two groups. Levels of miR-122 and miR-34a were positively correlated with disease severity from simple steatosis to steatohepatitis, supporting the potential value of these two miRNAs to serve as noninvasive biomarkers for NAFLD progression. Similarly, in an investigation of the relative expression of eight specific miRNAs (miR-197, miR-146b, miR-10b, miR-181d, miR-34a, miR-122, miR-99a, and miR-29a), serum levels of miR-181d, miR-99a, miR-197, and miR-146b were significantly lower in biopsy-proven NAFLD patients compared with healthy controls . Levels of miR-10b and miR-197 were inversely correlated with degree of inflammation, while miR-181d and miR-99a were inversely correlated with serum gamma 
glutamyl transferase (GGT) levels in NASH patients. However, the sample size was limited in this study (20 NAFLD patients and 20 controls), and validation of these findings in a larger study is warranted. In a study of miR-15b, which has been implicated in TNFmediated hepatocyte apoptosis, significantly higher circulating levels were found in 69 NAFLD patients compared to 42 healthy controls . These findings were consistent with those obtained in an animal model for NAFLD -high dietary fat-induced nonalcoholic fatty liver disease Sprague Dawley rats- and in a palmitate-induced NAFLD L02 cell culture model, with decreased cell proliferation, increased glucose consumption, and increased storage of intracellular triglycerides suggested as potential mechanisms. However, significant differences in BMI, blood glucose, triglyceride levels, total cholesterol, and ALT were present between the NAFLD and control groups; thus, additional studies will be necessary to demonstrate the robustness of miR-15b as a biomarker for human NAFLD. It is also worth noting that NAFLD patients were likely skewed toward severe disease given the criteria for persistently elevated aminotransferase levels, which we have found do not occur in most obese patients with biopsy-proven NAFLD .

A well-powered, multistage, case-control study was recently conducted to identify a serum miRNA profile as a surrogate marker for NAFLD . In that study, pooled serum samples from 20 NAFLD patients and 20 healthy controls were subjected to RNA-sequencing. Six miRNAs were significantly upregulated in NAFLD, including miR-122-5p, miR-1290, miR-27b-3p, miR-192-5p, miR-148a-3p, and miR-99a-5p. Of these, miR-122-5p, miR-1290, miR-27b-3p, and miR-192-5p were validated in an independent sample of 152 NAFLD patients and 90 unaffected controls. A stepwise logistic regression model was used to estimate the risk of being diagnosed with NAFLD using a training data set of 242 serum samples, and an independent data set comprised of 183 serum samples was used for validation. The AUC for the miRNA panel was 0.891 , suggesting potential clinical utility. Interestingly, the corresponding AUCs for patients with NASH Clinical Research Network Nash Activity Score (NAS) for stages $<3, \geq 3-<5$, and $\geq 5$ were $0.826,0.937$, and 0.860 , respectively, demonstrating that the diagnostic performance of the miRNA panel was greater with more active disease. In the same samples, the AUCs for alanine transaminase (ALT) and FIB-4 (a formula consisting of age, AST [aspartate transaminase], ALT, and platelets) were 0.786 and 0.795 , respectively, indicating that the miRNA panel had a higher sensitivity and specificity for NAFLD than either ALT or FIB-4. Compared with other studies on circulating miRNAs in diagnosing NAFLD, this study is unique because a large number of serum miRNAs were analyzed using deep sequencing, in contrast to the use of quantitative real-time PCR of individual miRNAs or PCR-based arrays, which provided an enhanced ability to identify potential diagnostic markers. We have found that next generation sequencing is a sensitive method for detecting low abundance transcripts and offers a powerful means with which to screen for both known and new miRNAs .

The identification of a serum miRNA panel that can differentiate NAFLD patients from healthy controls with a high degree of accuracy in a large number of participants suggests that miRNAs hold promise as reliable biomarkers. However, their potential utility is limited by a number of factors relevant to NAFLD, including the lack of cell-type specificity and the complexity of contributions from co-morbid conditions, particularly obesity, T2D, and dyslipidemia, with which a variety of miRNAs have been associated . Further, our 
knowledge of the potential value of circulating miRNAs to serve as biomarkers for NAFLD remains relatively limited. Yet current findings, combined with the rapidly expanding field of miRNA research, suggests that the identification and validation of circulating miRNA signatures may facilitate the development of improved methods for diagnosis and clinical monitoring of disease progression.

\section{References}

1. Demir M, Lang S, Steffen HM. Nonalcoholic fatty liver disease: Current status and future directions. J Dig Dis. 2015 Sep 25.

2. Satapathy SK, Sanyal AJ. Epidemiology and Natural History of Nonalcoholic Fatty Liver Disease. Semin Liver Dis. 2015 Aug; 35(3):221-35. [PubMed: 26378640]

3. Gerhard GSBP, Wood GC, Xin C, Argyrouplous G, Petrick A, Strodel WE, Gabrielson JD, Ibele A, Still CD, Kingsley C, DiStefano JK. Identification of Novel Clinical Factors Associated with Hepatic Fat Accumulation in Extreme Obesity. Journal of Obesity. 2014; 2014:1-8.

4. Sumida Y, Nakajima A, Itoh Y. Limitations of liver biopsy and non-invasive diagnostic tests for the diagnosis of nonalcoholic fatty liver disease/nonalcoholic steatohepatitis. World journal of gastroenterology : WJG. 2014 Jan 14; 20(2):475-85. [PubMed: 24574716]

5. Castera L, Pinzani M. Non-invasive assessment of liver fibrosis: are we ready? Lancet. 2010 Apr 24; 375(9724):1419-20. [PubMed: 20417845]

6. Kim DH, Saetrom P, Snove O Jr, Rossi JJ. MicroRNA-directed transcriptional gene silencing in mammalian cells. Proc Natl Acad Sci U S A. 2008 Oct 21; 105(42):16230-5. [PubMed: 18852463]

7. Gamazon ER, Innocenti F, Wei R, Wang L, Zhang M, Mirkov S, et al. A genome-wide integrative study of microRNAs in human liver. BMC genomics. 2013; 14:395. [PubMed: 23758991]

8. Szabo G, Bala S. MicroRNAs in liver disease. Nature reviews Gastroenterology \& hepatology. 2013 Sep; 10(9):542-52. [PubMed: 23689081]

9. Enache LS, Enache EL, Ramiere C, Diaz O, Bancu L, Sin A, et al. Circulating RNA molecules as biomarkers in liver disease. International journal of molecular sciences. 2014; 15(10):17644-66. [PubMed: 25272224]

10. Gilad S, Meiri E, Yogev Y, Benjamin S, Lebanony D, Yerushalmi N, et al. Serum microRNAs are promising novel biomarkers. PLoS One. 2008; 3(9):e3148. [PubMed: 18773077]

11. Zhang J, Li S, Li L, Li M, Guo C, Yao J, et al. Exosome and exosomal microRNA: trafficking, sorting, and function. Genomics Proteomics Bioinformatics. 2015 Feb; 13(1):17-24. [PubMed: 25724326]

12. Javidi MA, Ahmadi AH, Bakhshinejad B, Nouraee N, Babashah S, Sadeghizadeh M. Cell-free microRNAs as cancer biomarkers: the odyssey of miRNAs through body fluids. Med Oncol. 2014 Dec.31(12):295. [PubMed: 25362261]

13. Giordano S, Columbano A. MicroRNAs: new tools for diagnosis, prognosis, and therapy in hepatocellular carcinoma? Hepatology. 2013 Feb; 57(2):840-7. [PubMed: 23081718]

14. Gerhard GS, DiStefano JK. Micro RNAs in the development of non-alcoholic fatty liver disease. World J Hepatol. 2015 Feb 27; 7(2):226-34. [PubMed: 25729477]

15. Pirola CJ, Fernandez Gianotti T, Castano GO, Mallardi P, San Martino J, Mora Gonzalez, Lopez Ledesma M, et al. Circulating microRNA signature in non-alcoholic fatty liver disease: from serum non-coding RNAs to liver histology and disease pathogenesis. Gut. 2014 Jun 27.

16. Miyaaki H, Ichikawa T, Kamo Y, Taura N, Honda T, Shibata H, et al. Significance of serum and hepatic microRNA-122 levels in patients with non-alcoholic fatty liver disease. Liver Int. 2014 Aug; 34(7):e302-7. [PubMed: 24313922]

17. Yamada H, Ohashi K, Suzuki K, Munetsuna E, Ando Y, Yamazaki M, et al. Longitudinal study of circulating miR-122 in a rat model of non-alcoholic fatty liver disease. Clin Chim Acta. 2015 Jun 15.446:267-71. [PubMed: 25958847]

18. Hasegawa Y, Kim SR, Hatae T, Ohta M, Fujinami A, Sugimoto K, et al. Usefulness of Cytokeratin-18M65 in Diagnosing Non-Alcoholic Steatohepatitis in Japanese Population. Dig Dis. 2015 Oct; 33(6):715-20. [PubMed: 26488474] 
19. Joka D, Wahl K, Moeller S, Schlue J, Vaske B, Bahr MJ, et al. Prospective biopsy-controlled evaluation of cell death biomarkers for prediction of liver fibrosis and nonalcoholic steatohepatitis. Hepatology. 2012 Feb; 55(2):455-64. [PubMed: 21993925]

20. Thomas M, Deiters A. MicroRNA miR-122 as a therapeutic target for oligonucleotides and small molecules. Curr Med Chem. 2013; 20(29):3629-40. [PubMed: 23745562]

21. Cermelli S, Ruggieri A, Marrero JA, Ioannou GN, Beretta L. Circulating microRNAs in patients with chronic hepatitis C and non-alcoholic fatty liver disease. PLoS One. 2011; 6(8):e23937. [PubMed: 21886843]

22. Celikbilek M, Baskol M, Taheri S, Deniz K, Dogan S, Zararsiz G, et al. Circulating microRNAs in patients with non-alcoholic fatty liver disease. World J Hepatol. 2014 Aug 27; 6(8):613-20. [PubMed: 25232454]

23. Zhang Y, Cheng X, Lu Z, Wang J, Chen H, Fan W, et al. Upregulation of miR-15b in NAFLD models and in the serum of patients with fatty liver disease. Diabetes Res Clin Pract. 2013 Mar; 99(3):327-34. [PubMed: 23287814]

24. Petrick A, Benotti P, Wood GC, Still CD, Strodel WE, Gabrielsen J, et al. Utility of Ultrasound, Transaminases, and Visual Inspection to Assess Nonalcoholic Fatty Liver Disease in Bariatric Surgery Patients. Obes Surg. 2015 Dec; 25(12):2368-75. [PubMed: 26003548]

25. Tan Y, Ge G, Pan T, Wen D, Gan J. A pilot study of serum microRNAs panel as potential biomarkers for diagnosis of nonalcoholic fatty liver disease. PloS one. 2014; 9(8):e105192. [PubMed: 25141008]

26. Leti F, Malenica I, Doshi M, Courtright A, Van Keuren-Jensen K, Legendre C, et al. Highthroughput sequencing reveals altered expression of hepatic microRNAs in nonalcoholic fatty liver disease-related fibrosis. Transl Res. 2015 Sep; 166(3):304-14. [PubMed: 26001595]

27. Abente EJ, Subramanian M, Ramachandran V, Najafi-Shoushtari SH. MicroRNAs in obesityassociated disorders. Arch Biochem Biophys. 2015 Sep 26. 